\title{
The Value of Speckle Tracking Strain Imaging at Recovery Period of Dobutamine Stress Echocardiography
}

\author{
Mosaad M. Abushabana', Neveen I. Samy², Mahmoud A. Soliman², Walaa Fareed ${ }^{2}$ \\ ${ }^{1}$ El Mahalla General Hospital, MOH, El-Mahalla, Egypt \\ ${ }^{2}$ Cardiovascular Disease Department, Faculty of Medicine, Menoufia University, Shebeen El-Kom, Egypt \\ Email: neveinsami@yahoo.com
}

How to cite this paper: Abushabana, M.M., Samy, N.I., Soliman, M.A. and Fareed, W. (2019) The Value of Speckle Tracking Strain Imaging at Recovery Period of Dobutamine Stress Echocardiography. World Journal of Cardiovascular Diseases, 9, 223-235. https://doi.org/10.4236/wjcd.2019.93020

Received: February 14, 2019

Accepted: March 22, 2019

Published: March 25, 2019

Copyright $\odot 2019$ by author(s) and Scientific Research Publishing Inc. This work is licensed under the Creative Commons Attribution International License (CC BY 4.0).

http://creativecommons.org/licenses/by/4.0/

\begin{abstract}
Background: Dobutamine stress echocardiography (DSE) is a well-established method for detecting myocardial ischemia. The classic echocardiographic findings of ischemia are new or worsening abnormalities of radial wall thickening. However, interpretation of these findings is subjective and dependent on experience. Speckle tracking strain imaging (STI) has been introduced as a novel method to overcome these limitations. Objective: The aim of the study is to evaluate the value of speckle tracking imaging (STI) by estimating the mean global longitudinal systolic (GLS) and the mean global circumferential systolic (GCS) strain imaging at recovery period of dobutamine stress echocardiography for prediction of significant coronary artery disease (CAD) in patients with chest discomfort. Methods: Fifty three patients presented for evaluation of chest discomfort underwent dobutamine stress echocardiography and coronary angiography at Cardiology Department, Menoufia University Hospital. The mean global left ventricular longitudinal systolic strain (GLS) at apical views -3 chambers, 4 chambers $\& 2$ chambers apical views and global left ventricular circumferential systolic strain (GCS) at short axis view at level of papillary muscle were measured at rest and during recovery period of dobutamine stress echocardiography using automated functional imaging (AFI). Coronary angiography was done for all patients. Significant coronary artery lesion was defined as having a $\geq 70 \%$ diameter stenosis on coronary angiography. Patients were divided into two groups based on the presence or absence of significant coronary artery lesion into CAD positive (+ve) group vs. CAD negative (-ve) group. Results: In both groups, there were no statistically significant differences in the clinical characteristics and baseline conventional transthoracic echocardiography. GLS and GSC at recovery were lower in the CAD (+ve) group than in the CAD (-ve) group $(-16.69 \% \pm 1.10 \%$ vs
\end{abstract}


$-19.05 \% \pm 1.41 \% \mathrm{p}<0.0001$ respectively for GLS and $-19.90 \pm 1.69$ vs $-21.24 \% \pm 1.49 \% \mathrm{p}=0.002$ respectively for GCS). GLS and GCS during recovery period of DSE were valuable for prediction of CAD when cutoff at recovery is less than $-17.95 \%$ (sensitivity of $84 \%$ \& specificity of $84 \%$ ) for GLS and less than $-20.2 \%$ (sensitivity of $76 \%$ \& specificity of $84 \%$ ) for GCS. Also, we observed that the GLS and GCS percentile decreased from baseline to recovery period of DSE by about $-9.95 \% \pm 4.82 \%$ and $-7.72 \% \pm 3.90 \%$ respectively. Conclusion: Speckle tracking Imaging GLS and GCS at recovery period of Dobutamine Stress Echocardiography is feasible and offers an objective technique for prediction of significant coronary artery disease with increasing the accuracy of DSE in patient with chest discomfort.

\section{Keywords}

Coronary Artery Disease, Dobutamine Stress Echocardiography, Speckle Tracking Imaging, Coronary Angiography

\section{Introduction}

Coronary artery disease (CAD) is a major cause of death and disability in developed countries. Although CAD mortality rates have declined over the past four decades in the United States (and elsewhere), CAD remains responsible for about one-third of all deaths in individuals over age 35 [1].

The combination of stress testing and echocardiography-SEcho-has assumed an important role in the diagnosis of CAD. The appearance of a new stress-induced regional wall motion abnormality (WMA), readily identified by echocardiography, allows the detection of ischemia, and its location may be used to predict the stenosis in coronary vessels [2]. Global and regional cardiac functions are key parameters reported during this test. Their assessment is mostly based on visual interpretation of the B-mode cine loop with a high risk of intraand inter-observer variability, depending on the reader's experience [3].

The accuracy of stress echo tests for the detection of coronary artery disease is expressed as the sensitivity and specificity of the technique for the detection of angiographically demonstrated stenosis. Nonetheless, this parameter has a number of limitations, some reflecting the limitations of an angiographic cutoff for significant disease, including the variation of the physiologic effect of a stenosis based on site, length, and vessel size, as well as over- and under-estimation of coronary lesion severity [4].

Speckle tracking imaging (STI) is a newer approach to assess myocardial deformation [5]. It relies on detecting features (mostly "speckles") in 2D greyscale images, the displacement of which is measured. Velocity, strain and strain rate is then derived from the displacement information. Speckles can be tracked in any direction within the image plane which offers new options for motion and deformation analysis compared to the one-dimensional tissue Doppler imaging 
(TDI) method [6]. Assessment of STI at peak stress of DSE is especially difficult because of an inadequate low frame rate for the rapid heart rate, failure to maintain respiratory holding in symptomatic patients, subsequent tracking problems and the development of signal to noise due to hyper dynamic LV contractility and excessive annular motion. While assessment of STI at recovery is easier because of the ease of respiratory control due to improvement in symptoms, lower heart rate and decreased myocardial hyper-contractility [7]. So this study aimed to evaluate the value of speckle tracking imaging during the recovery period after DSE for prediction of significant CAD in patients with chest discomfort.

\section{Methods}

This prospective study was carried on 53 patients who presented with chest pain to cardiac clinic at Menoufia university hospital during the period between June 2017 and July 2018. While patients with acute coronary syndrome, wall motion abnormalities (WMAs) at rest or previous myocardial infarction, previous cardiac surgery, valvular heart disease of greater than moderate grade, Non-sinus rhythm such as atrial fibrillation, Artificial pacemaker, Systolic dysfunction with left ventricular (LV) ejection fraction $(\mathrm{EF})<50 \%$, patient with Systemic diseases including chronic obstructive pulmonary syndrome and also patients with poor echogenic window were excluded from the analysis. All patients underwent dobutamine stress echocardiography and coronary angiography after giving written informed consent.

\subsection{Dobutamine Stress Echocardiography and Speckle Tracking Imaging}

Patients underwent dobutamine stress echocardiography, the examinations were performed in the left lateral supine position with Vivid E9 machine (GE Vingmed, Norway) using standard protocol with incremental infusion of 5, 10, 20,30 and $40 \mathrm{microgram} / \mathrm{kg} / \mathrm{min}$ every 3 minutes and up to $1 \mathrm{mg}$ of atropine if the target heart rate not achieved ( $85 \%$ of the age predicted maximal heart rate).

Heart rate, blood pressure, 12 leads electrocardiogram and symptoms during the test were recorded. Beta blockers were kept in hold 2 days at least before the test. Criteria for termination the test were completion of the protocol, symptomatic hypotension, severe chest pain, and increase systolic blood pressure more than $220 \mathrm{mmhg}$, diastolic blood pressure more than $120 \mathrm{mmhg}$, serious supraventricular or ventricular arrhythmias [8]. Estimate left ventricular systolic function \& Wall motion visually using 17 myocardial segment models at each DSE [9].

At frame rate between 40 to 70 [10] frame per second 2 Dimensional gray-scale cine loops from three- plane strain study at apical-3 chamber, 4 chamber \& 2 chamber-views and at short axis view at level of papillary muscle, were acquired in the end-expiratory holding state at rest and during recovery period of dobutamine stress echocardiography. U-shaped LV myocardium mean 
global left ventricular longitudinal systolic strain (GLS) and circle shaped global left ventricular circumferential systolic strain (GCS) were measured as follows: global strain $\%=\mathrm{L}$ (end-systolic) $-\mathrm{L}$ (end-diastolic)/L (end-diastolic) $\times 100$ [11] [12], using automated functional imaging (AFI) with tracing of the endocardium border, and adjusted region of interest (ROI) to include the entire myocardium at end-systolic frame at aortic valve closure (AVC) [13]. All images analyzed offline used Echo PAC SW version 9.0.0, GE Vingmed Ultrasound Horton Norway).

\subsection{Coronary Angiography}

Coronary angiography was performed for all patients utilizing retrograde percutaneous trans-radial and trans-femoral technique for evaluation of the coronary lesion. Conventional views with addition cranial and caudal angulations were used to maximize imaging of coronary lesions. According to coronary angiography findings patients were divided into two groups. Group 1: CAD (+ve): including patients with significant CAD including coronary artery lesion $>70 \%$ diameter stenosis (assessed by visual inspection) [14] and group 2: CAD (-ve): including patients without significant CAD including normal coronaries defined as no luminal narrowing and non-significant lesion is defined as CAD lesion having less than $40 \%$ by coronary angiography [15]. Patients with intermediate coronary artery lesions between $40 \% \& 70 \%$ were excluded as FFR to evaluate the significance of the lesion was not available.

All data were exported to a spread-sheet (Microsoft excel 2013, Microsoft Corporation, Redmond, WA, USA).

\subsection{Statistical Analysis}

Data were analyzed using SPSS (Statistical Package for Social Sciences), Qualitative variable were presented as number of Chi square or Fisher's exact test was used for comparison between groups, as appropriate. Qualitative variable were presented as mean + SD (Standard Deviation). Student t-test was used for comparison between groups. $\mathrm{P}$ value $<0.05$ was considered statistically significant [16]. Also the Mann Whitney test (U) (Non Parametric test) which is a test of significance used for comparison between two groups not normally distributed having quantitative variables. The optimal GLS \& CS cutoff values for detecting Coronary artery disease at baseline \& recovery period of DSE were determined from receiver operating characteristic (ROC) curves. The area under the ROC curve (AUC) was used to compare the diagnostic validity, and z-score was calculated to determine the difference of AUC [17].

\section{Results}

The 53 patients had mean age of $55.1 \pm 7.8$ years ( 31 male $58.5 \%$ and 22 female 41.5\%). Patients underwent DSE with the target heart rate was achieved in 47 patients (88.7\%). The remaining 6 patients were terminated because of ischemic 
signs. Patient classified into two groups based on the coronary angiography results, group 1: Coronary artery disease positive $(\mathrm{CAD}+\mathrm{ve})$ including 25 patients (47.2\%) and group 2: Coronary artery disease negative (CAD -ve) including 28 patients (52.8\%).

There was no significant difference as regards demographic data and risk factors between two groups (Table 1) and this actually was planned from the start to eliminate the possible effects of diabetes mellitus and hypertension on myocardial deformation proved by other studies [18] [19].

There is no significance difference between the two groups as regard systolic and diastolic blood pressure at rest or at peak DSE, only resting heart rate is higher in CAD +ve group than CAD -ve group ( $p=0.001$ ) (Table 1$)$.

No significance difference as regard baseline conventional transthoracic echocardiography including left ventricular (LV) size, LV wall thickness, ejection fraction (EF), left atrial (LA) size, volume and different diastolic parameter between the two groups (Table 2).

Wall motion abnormalities (WMA) at peak DSE were observed in 21 patients $(84 \%)$ in the CAD +ve group and in 6 patients $(21.4 \%)$ in the CAD -ve group with a sensitivity and specificity of WMA for CAD of $84 \%$ and $84.6 \%$, respectively. WMA were normalized at recovery period of DSE (Table 3).

In CAD +ve patients, the values of GLS and GCS at recovery period of DSE had significantly greater reduction $(\mathrm{p}<0.0001, \mathrm{p}=0.002$ respectively) when compared with GLS and GSC at same stage of DSE in CAD -ve individuals respectively. While there is no significant difference between the two studied groups as regard the GLS and GCS at rest (Table 4). With NPar Tests, Mann-whitney Test the GLS and GCS percentile change from baseline to recovery period of DSE $=-9.95 \% \pm 4.82 \% \mathrm{p}<0.0001$ and $-7.72 \% \pm 3.90 \% \mathrm{p}=0.001$ respectively (Table 5). Upon ROC analysis, the values of GLS and GCS at recovery period of DSE showed significantly greater AUCs than the values of GLS and GCS at rest respectively $(\mathrm{p}<0.0001, \mathrm{CI}=95 \%$ and $\mathrm{p}=0.002, \mathrm{CI}=95 \%)$ respectively. The optimal cutoff value of GLS and GCS at recovery for prediction of significant CAD was $-17.95 \%$ and $-20.2 \%$ respectively, which resulted in a GLS sensitivity and specificity of $84 \%$ and $84 \%$ respectively (Figure 1), and GCS sensitivity and specificity of $76 \%$ and of $83.5 \%$ respectively (Figure 2 ). There was no significant difference as regard sensitivity, specificity and accuracy values between visual WMA at peak stress and the quantitative GLS (Table 6) and GCS (Table 7) technique at recovery period of DSE.

\section{Discussion}

Although stress ECHO is a relatively easy to use technology that poses only a low risk of adverse events compared to other imaging technologies, it may potentially be overused and/or misused in CAD diagnosis [20]. Several recent advances have been made focusing on quantitative methods for assessment, improved image quality and enhanced portability, however, evidence on the effectiveness and clinical utility of these enhancements is limited [21]. 
Table 1. Baseline characteristics between CAD positive patients and CAD negative individuals.

\begin{tabular}{cccc}
\hline & CAD (+) $\mathbf{n}=\mathbf{2 5}$ & CAD (-) $\mathbf{n = 2 8}$ & p value \\
\hline Male n (\%) & $14(56 \%)$ & $17(60.7 \%)$ & 0.761 \\
Age (yrs.) & $55.52 \pm 7.19$ & $54.57 \pm 8.37$ & 0.668 \\
Smoking n (\%) & $9(36)$ & $11(39)$ & 0.781 \\
HTN n (\%) & $16(64)$ & $14(50)$ & 0.286 \\
DM n (\%) & $17(68)$ & $16(57.1)$ & 0.348 \\
Dyslipidemia n (\%) & $17(68)$ & $15(53.57)$ & 0.317 \\
Hear rate b/min & $76.16 \pm 5.27$ & $68.47 \pm 4.25$ & 0.001 \\
Baseline SBP & $135.28 \pm 6.46$ & $132.92 \pm 8.16$ & 0.263 \\
Baseline DBP & $77.2 \pm 8.13$ & $79.75 \pm 4.45$ & 0.174 \\
BMI (Kg/m ${ }^{2}$ ) & $28.17 \pm 2.09$ & $27.69 \pm 1.77$ & 0.385 \\
\hline
\end{tabular}

n: number of cases , CAD: coronary artery disease, HTN: hypertension, DM: diabetes mellitus, SBP: systolic blood pressure, DBP: diastolic blood pressure, BMI: body mass index.

Table 2. Baseline transthoracic echocardiography between CAD Positive and CAD negative group.

\begin{tabular}{cccc}
\hline Variable & CAD $(+) \mathbf{n}=\mathbf{2 5}$ & CAD $(-) \mathbf{n}=\mathbf{2 8}$ & p value \\
\hline LVDd mm & $44.12 \pm 2.92$ & $44 \pm 3.29$ & 0.892 \\
LVDs mm & $30.36 \pm 1.91$ & $31.24 \pm 1.81$ & 0.101 \\
IVS mm & $10.44 \pm 1.42$ & $10.68 \pm 1.44$ & 0.555 \\
PW mm & $10.64 \pm 1.5$ & $10.64 \pm 1.08$ & 1.000 \\
EF\% & $61.56 \pm 4.24$ & $61.92 \pm 4.28$ & 0.766 \\
LA mm & 30.16 & 31.36 & 0.249 \\
LAVI ml/m ${ }^{2}$ & $24.56 \pm 4.2$ & $24.1 \pm 3.57$ & 0.485 \\
E/A ratio & $0.95 \pm 0.12$ & $0.95 \pm 0.13$ & 0.946 \\
IVRT mm & $84.72 \pm 4.2$ & $83.56 \pm 3.57$ & 0.298 \\
E/e' & $8.44 \pm 1.23$ & $8.94 \pm 1.16$ & 0.146 \\
\hline
\end{tabular}

Data is expressed as mean \pm standard deviation. $\mathrm{n}$ : number of cases, CAD: coronary artery disease, LVDd: left ventricular diastolic dimension, LVDs: left ventricular systolic dimension, IVS: interventricular septal thickness, PW: end-diastolic left ventricular posterior wall thicknesses, EF: ejection fraction, LA: left atrial dimension, LAVI: left atrial volume index, IVRT: isovolumic relaxation time E: early diastolic transmitral velocity, A: late diastolic transmitral velocity, $\mathrm{E}^{\prime}$ : early diastolic mitral annular velocity.

Speckle tracking, a tool based on gray-scale image processing emerged as an exciting alternative for assessing global and regional myocardial deformations. Speckle tracking analysis is based on a block-matching approach of the specklepatterns within the myocardium. It allows strain assessment of myocardial segments relatively independent of their position with respect to the ultrasound beam direction [22] [23].

Recent studies showed that transient ischemic injury after DSE persists and can persist for at least 5 minutes in $90 \%$ of patients [22] [25]. According to 
Table 3. WMA at peak of DSE in CAD positive and CAD negative individual's patients.

\begin{tabular}{lccccc}
\hline & \multicolumn{2}{c}{ CAD positive } & \multicolumn{2}{c}{ CAD negative } & p value \\
\cline { 2 - 6 } & $\mathrm{n}$ & $\%$ & $\mathrm{n}$ & $\%$ & \\
\hline WMA (present) & 21 & 84 & 6 & 21.4 & $<0.0001$ \\
WMA (absent) & 4 & 16 & 22 & 78.6 & \\
\hline
\end{tabular}

n: number of cases, WMA wall motion abnormalities, CAD: Coronary artery disease.

Table 4. Comparison between CAD negative individuals and CAD positive patients as regard Global Longitudinal Strain (GLS) and Global Circumferential Strain (GCS) at rest and at recovery period during dobutamine Stress Echocardiography.

\begin{tabular}{ccccc}
\hline & & CAD positive & CAD negative & p value \\
\hline GLS at rest & Mean \pm SD & $-18.54 \pm 1.36$ & $-18.92 \% \pm 1.43 \%$ & 0.273 \\
GLS at Recovery of DSE & Mean \pm SD & $-16.69 \pm 1.10$ & $-19.05 \pm 1.41$ & $<0.0001$ \\
GCS at rest & Mean \pm SD & $-21.24 \% \pm 1.49 \%$ & $-21.59 \% \pm 1.25 \%$ & 0.885 \\
GCS at recovery period of DSE & Mean \pm SD & $-19.90 \% \pm 1.69 \%$ & $-21.71 \% \pm 1.49 \%$ & 0.002 \\
\hline
\end{tabular}

DSE: Dobutamine Stress Echocardiography, GLS: Global Longitudinal strain, GCS: Global Circumferential strain, CAD: coronary artery disease.

Table 5. Percentage of change in GLS and GCS measures from baseline to recovery.

\begin{tabular}{ccc}
\hline & $\begin{array}{c}\text { Percentage of change in GLS } \\
\text { measure from } \\
\text { baseline to recovery } \\
\text { Mean } \pm \text { SD }\end{array}$ & $\begin{array}{c}\text { Percentage of change in GCS } \\
\text { measure from } \\
\text { baseline to recovery }\end{array}$ \\
p value & $-9.95 \% \pm 4.82 \%$ & $-7.72 \% \pm 3.90 \%$ \\
& $<0.0001$ & 0.001 \\
\hline
\end{tabular}

GLS: Global longitudinal strain, GCS: Global Circumferential strain.

Table 6. Sensitivity, specificity and accuracy of wall motion abnormalities at peak stress and GLS value lesser than $-17.95 \%$ at recovery period of DSE for prediction of significant CAD.

\begin{tabular}{cccc}
\hline & WMA + ve at Peak stress & GLS at Recovery DSE $<-17.95 \%$ & p value \\
\hline Sensitivity & $84 \%$ & $84 \%$ & 1.000 \\
Specificity & $84.6 \%$ & $84 \%$ & 0.963 \\
Accuracy & $79.2 \%$ & $77.4 \%$ & 0.886 \\
\hline
\end{tabular}

WMA: wall motion abnormality, GLS: Global left ventricular strain, DSE: dobutamine stress echocardiography.

Hui-Jeong Hwang et al. [26] suggest that systolic stunning after a transient ischemic event persists and that assessment by GLS during the recovery period allows this phenomenon to be objectively and quantitatively assessed.

This study was designed to investigate whether assessment of myocardial deformation using STI during the recovery period after DSE allows for detection of CAD in patients with chest discomfort. GLS \& GCS at recovery period of DSE may be a good marker for the prediction of significant CAD, as well as developed WMA at peak stress similar result from Patricia et al. [27] showed that 
Table 7. Sensitivity, specificity and accuracy of wall motion abnormalities at peak stress and GCS value lesser than $-20.02 \%$ at recovery period of DSE for prediction of significant CAD.

\begin{tabular}{cccc}
\hline & WMA +ve at peak stress & GCS at recovery DSE $<-20.2 \%$ & p value \\
\hline Sensitivity & $84 \%$ & $76 \%$ & 0.527 \\
Specificity & $84.6 \%$ & $83.5 \%$ & 0.969 \\
Accuracy & $79.2 \%$ & 67.9 & 0.351 \\
\hline
\end{tabular}

WMA: wall motion abnormality, DSE: dobutamine stress echocardiography, GCS: global circumferential strain.

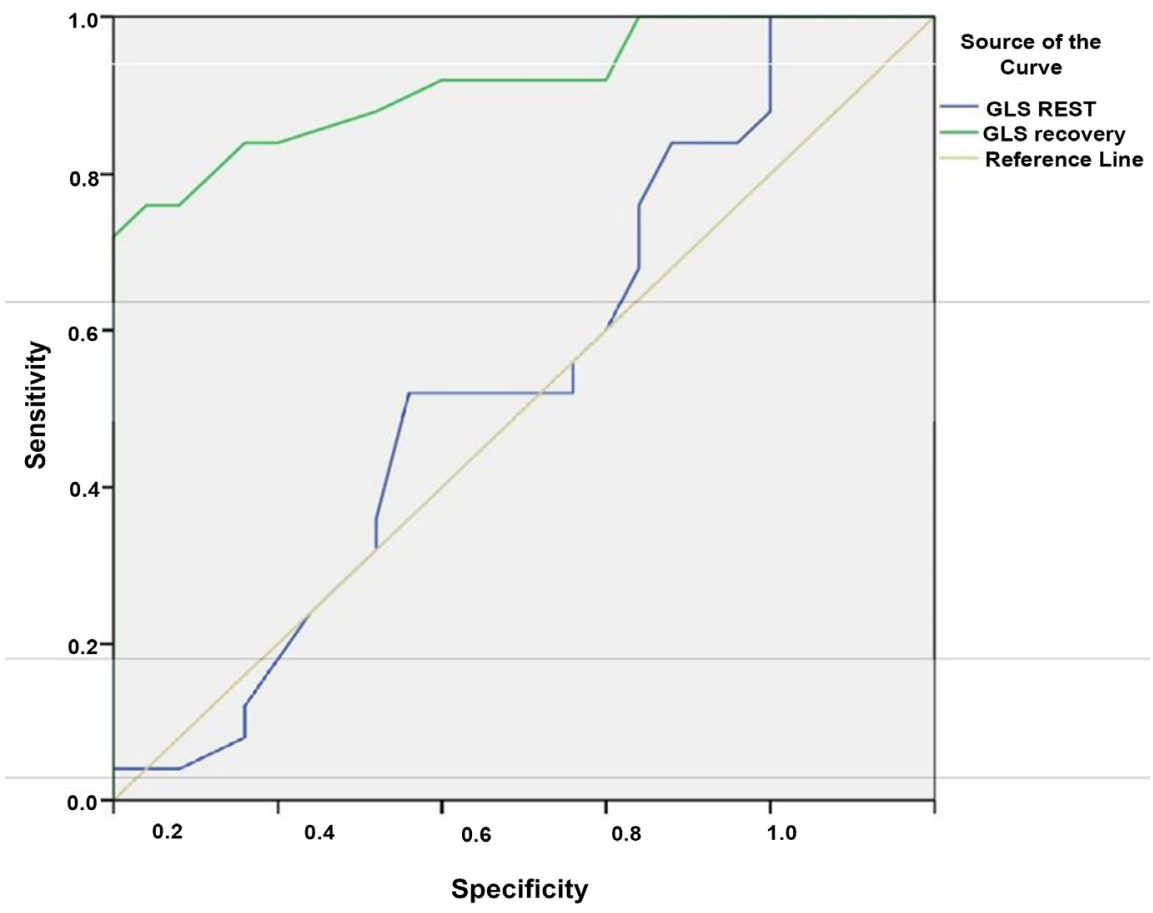

Figure 1. Reciever-operating characteristic curve of GLS at rest \& during recovery period of DSE for prediction of CAD. a sensitivity of $84 \%$ \& specificity of $84 \%$ are observed when cutoff at recovery is less than $-17.95 \%$ for prediction of CAD. GLS: Global Left ventricular Strain. — GLS at rest 0.547 (CI $0.385-0.710$ ); — GLS at recovery 0.913 (CI 0.832 - 0.993); Reference Line.

Evaluation of circumferential and longitudinal strains during DSE has real potential for quantitative evaluation of LV deformation in the routine assessment of ischemia.

The findings of our study demonstrate that measurement of speckle tracking imaging of both GLS and GCS during recovery period of dobutamine stress echocardiography is feasible and provide quantifiable information for the prediction of significant coronary artery disease. Our feasibility and reproducibility rates match those reported by other authors, Ryo $\mathrm{K}$ et al. [28] who shown that evaluation of circumferential and longitudinal strains during DSE has real potential for quantitative evaluation of LV deformation in the routine assessment of ischemia. 


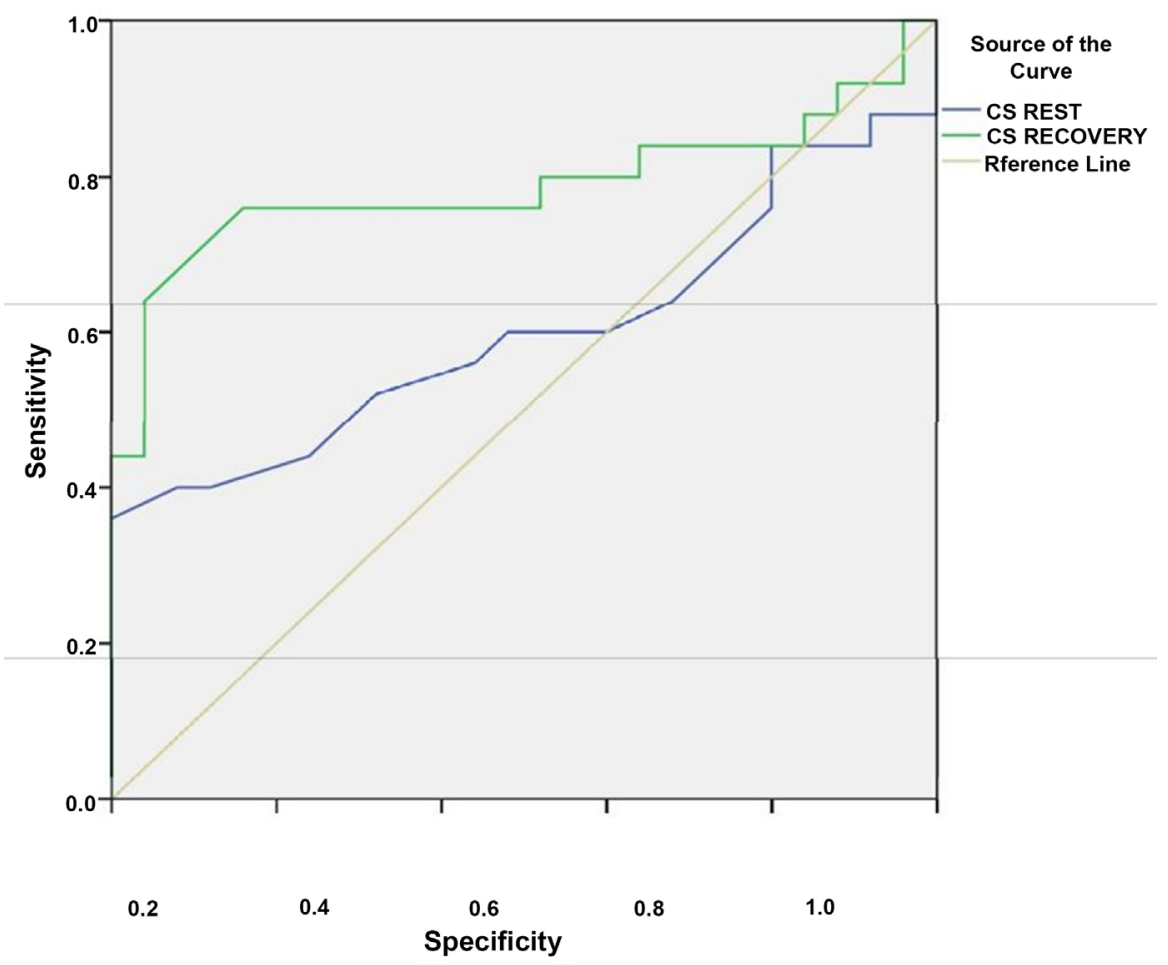

Figure 2. CS at rest 0.547 (CI $0.435-0.765) \ldots$ CS at recovery 0.913 (CI $0.648-0.929$ ) reciever-operating characteristic curve of CS at rest \& during recovery period of DSE for prediction of CAD. A sensitivity of $76 \%$ \& specificity of $83.5 \%$ are observed when cutoff at recovery is less than $-20.2 \%$ for prediction of CAD. CS: Circumferential strain. CS at rest 0.547 (CI $0.385-0.710$ ); — CS at recovery 0.913 (CI $0.832-0.993$ ); Reference Line.

We found that GLS \& GCS at recovery period of DSE may be a good marker for the prediction of significant CAD, as well as development of WMA at peak stress. Wierzbowska-D et al. [29] and Yu Y et al. [30] found that 2D strain can be calculated at any stage of DSE giving significant information for the presence of ischemia, even at low dose stage.

This study has revealed that longitudinal strain had higher diagnostic accuracy than circumferential strains and reported similar diagnostic accuracy as expert wall motion analysis for the detection of $\mathrm{CAD}$, this finding is match report with other author, Ng AC et al. [7] found that Longitudinal strain analysis had comparable accuracy to wall motion score index (WMSI) and its diagnostic accuracy was incremental to either longitudinal strain or WMSI alone and they concluded that the Longitudinal strain analysis had higher diagnostic accuracy than circumferential and radial strains and was comparable to WMSI for detection of significant coronary artery disease. Constantina A. et al. [31] who found that the combination of GLS and WMSI offers enhanced diagnostic ability. Valttri U et al. [32] who showed that GLS, strain rate and Post systolic strain index (PSI) spickle tracking echocardiography during early recovery after DSE can help in detection of hemodynamically significant coronary artery disease compared with visual wall motion alone. 
In this study, the sensitivity of newly developed WMA at peak stress for detection of CAD was similar to Hui-Jeong Hwang, et al. [26].

In this study the amplitude cutoffs of GLS \& GCS for the prediction of significant CAD at recovery period of DSE is lower when compared with rest with high diagnostic accuracy, similar result from Hui-Jeong Hwang, et al. [26] for GLS, Patricia et al. [27] for CS. Therefore, the values of speckle tracking Imaging GLS and GCS at recovery period of DSE may be offer useful method for prediction of significant CAD and added to the test to aid inexperienced interpreter of wall motion analysis.

\section{Study Limitations}

The strain rate was not analyzed in fact, and the noise in the strain rate signal increased with higher heart rates, and the image quality decreased. Second, we only showed global longitudinal strain analysis using an automatic functional imaging method. Another limitation is that this was a study of relatively small sample size.

\section{Conclusion}

Speckle tracking imaging with estimation of GLS and GCS at recovery period of Dobutamine Stress Echocardiography is feasible and offers an objective technique for prediction of significant coronary artery disease with increasing the accuracy of DSE in patients with chest discomfort.

\section{Conflicts of Interest}

The authors declare no conflicts of interest regarding the publication of this paper.

\section{References}

[1] Sanchis-Gomar, F., Perez-Quilis, C., Leischik, R. and Lucia, A. (2016) Epidemiology of Coronary Heart Disease and Acute Coronary Syndrome. Annals of Translational Medicine, 4, 256. https://doi.org/10.21037/atm.2016.06.33

[2] Cortez, N. and Pinto, D.F.J. (2009) Stress Echocardiography in Diagnosis of Coronary Artery Disease. US Cardiology, 6, 37-42.

[3] Picano, E., Lattanzi, F., Orlandini, A., Marini, C. and L’Abbate, A. (1991) Stress Echocardiography and the Human Factor: The Importance of Being Expert. Journal of the American College of Cardiology, 17, 666-669.

[4] Sothinathan, G., Grace, Y., Guy, P., Nikos, K., Anastasia, V., Ahmed, E. and Roxy, S. (2016) Diagnostic Accuracy of Stress Echocardiography Compared with Invasive Coronary Angiography with Fractional Flow Reserve for the Diagnosis of Haemodynamically Significant CAD in Patients with Known or Suspected CAD. https://doi.org/10.1136/heartjnl-2016-309890.132

[5] Leitman, M., Lysyansky, P., Sidenko, S., Shir, V., Peleg, E., Binenbaum, M., Kaluski, E., Krakover, R. and Vered, Z. (2004) Two-Dimensional Strain-A Novel Software for Real-Time Quantitative Echocardiographic Assessment of Myocardial Function. Journal of the American Society of Echocardiography, 17, 1021-1029. 
https://doi.org/10.1016/j.echo.2004.06.019

[6] Mada, R.O., Duchenne, J. and Voigt, J.-U. (2014) Tissue Doppler, Strain and Strain Rate in Ischemic Heart Disease "How I Do it". Cardiovascular Ultrasound, 12, 38. https://doi.org/10.1186/1476-7120-12-38

[7] Ng, A.C., Sitges, M., Pham, P.N., Tran Da, T., Delgado, V., Bertini, M., Nucifora, G., Vidaic, J., Allman, C., Holman, E.R., Bax, J.J. and Leung, D.Y. (2009) Incremental Value of 2-Dimensional Speckle Tracking Strain Imaging to Wall Motion Analysis for Detection of Coronary Artery Disease in Patients Undergoing Dobutamine Stress Echocardiography. American Heart Journal, 158, 836-844. https://doi.org/10.1016/j.ahj.2009.09.010

[8] Rallis's, L.S., Moisakos, I.E. and Nihoyannopoulos, P. (1998) Hypotensive Response during Dobutamine Stress Echocardiography in Coronary Patients: A Common Event of Well-Functioning Left Ventricle. Clinical Cardiology, 21, 747-752. https://doi.org/10.1002/clc.4960211010

[9] Szymanski, C., Pierard, L. and Lancellotti, P. (2011) Imaging Techniques in Coronary Atherosclerotic Disease: Dobutamine Stress Echocardiography-Evidence and Perspectives. Journal of Cardiovascular Medicine (Hagerstown), 12, 543-553. https://doi.org/10.2459/JCM.0b013e32834853f8

[10] Yingchoncharroen, T., Agarwal, S., Popovic, Z.B. and Marwick, T.H. (2013) Normal Ranges of Left Ventricular Strain: A Meta-Analysis. Journal of the American Society of Echocardiography, 26, 185-191.

[11] Reisner, S.A., Lysyansky, P., Agmon, Y., Mutlak, D., Lessick, J. and Friedman, Z. (2004) Global Longitudinal Strain: A Novel Index of Left Ventricular Systolic Function. Journal of the American Society of Echocardiography, 17, 630-633. https://doi.org/10.1016/j.echo.2004.02.011

[12] Cho, G.Y., Marwick, T.H., Kim, H.S., Kim, M.K., Hong, K.S. and Oh, D.J. (2009) Global 2-Dimentional Strain as a New Prognosticator in Patients with Heart Failure. Journal of the American College of Cardiology, 54, 618-624. https://doi.org/10.1016/j.jacc.2009.04.061

[13] Davis, A.M., Adams, D., Venkatesshvaran, A. and Alenezi, F. (2017) Speckle Tracking Strain Echocardiography: What Sonographer Need to Know! Journal of the Indian Academy of Echocardiography \& Cardiovascular Imaging, 1, 133-139.

[14] Li, J., Elrashidi, M.Y., Flammer, A.J., Lennon, R.J., Bell, M.R., Holmes, D.R., Bresnahan, J.F., Rihal, C.S., Lerman, L.O. and Lerman, A. (2013) Long-Term Outcomes of Functional Flow Reserve-Guided vs. Angiography-Guided Percutaneous Coronary Intervention in Contemporary Practice. European Heart Journal, 34, 1375-1383. https://doi.org/10.1093/eurheartj/eht005

[15] Tobis, J., Azarbal, B. and Slavin, L. (2007) Assessment of Intermediate Severity Coronary Lesions in the Catheterization Laboratory. JACC, 49, 839-848.

[16] Dawson-Sanders, B. and Trapp, R. (1994) Basic and Clinical Biostatistics. 2nd Edition, Lange Medical Book, Practice-Hall International Inc.

[17] DeLong, E.R., DeLong, D.M. and Clarke-Pearson, D.L. (1988) Comparing the Areas under Two or More Correlated Receiver Operating Characteristic Curves: A Nonparametric Approach. Biometrics, 44, 837-845. https://doi.org/10.2307/2531595

[18] Elgohary, A.A., Shalaby, M.A., Mahfouz, R.A. and Mohamed, M.G. (2017) Effect of Diabetic Duration on Left Vntricular Longitudinal Strain by Speckle Tracking Imaging. $A J C, 119$, e6.

[19] Soufi Taleb Bendiab, N., Meziani Tani, A., Ouabdesselam, S., Methia, N., Latreche, 
S., Henaoui, L., Monseuz, J.J. and Benkhedda, S. (2017) Factors Associated with Global Longitudinal Strain Decline in Hypertensive Patients with Normal Left Ventricular Ejection Fraction. European Journal of Preventive Cardiology, 24, 1463-1472.

[20] Arbab-Zadeh, A. (2012) Stress Testing and Non-Invasive Coronary Angiography in Patients with Suspected Coronary Artery Disease: Time for a New Paradigm. Heart Internet, 7, $\mathrm{e}$.

[21] Peteiro, J. and Bouzas-Mosqueras, A. (2010) Exercise Echocardiography. World Journal of Cardiology, 2, 223-232. https://doi.org/10.4330/wjc.v2.i8.223

[22] Perk, G., Tunick, P.A. and Kronzon, I. (2007) Non-Doppler Two-Dimensional Strain Imaging by Echocardiography-From Technical Considerations to Clinical Applications. Journal of the American Society of Echocardiography, 20, 234-243.

[23] Helle-Valle, T., Crosby, J., Edvardsen, T., Lyseggen, E., Amundsen, B.H., Smith, H.-J., et al. (2005) New Noninvasive Method for Assessment of Left Ventricular Rotation: Speckle Tracking Echocardiography. Circulation, 112, 3149-3156.

[24] Tsoukas, A., Ikonomidis, I., Cokkinos, P. and Nihoyannopoulos, P. (1997) Significance of Persistent Left Ventricular Dysfunction during Recovery after Dobutamine Stress Echocardiography. Journal of the American College of Cardiology, 30, 621-626. https://doi.org/10.1016/S0735-1097(97)00226-X

[25] Ishii, K., Suyama, T., Imai, M., Maenaka, M., Yamanaka, A., Makino, Y., Seino, Y., Shimada, K. and Yoshikawa, J. (2009) Abnormal Regional Left Ventricular Systolic and Diastolic Function in Patients with Coronary Artery Disease Undergoing Percutaneous Coronary Intervention: Clinical Significance of Post-Ischemic Diastolic Stunning. Journal of the American College of Cardiology, 54, 1589-1597.

https://doi.org/10.1016/j.jacc.2009.06.030

[26] Hwang, H.-J., et al. (2014) The Value of Assessing Myocardial Deformation at Recovery after Dobutamine Stress Echocardiography. Journal of Cardiovascular UItrasound, 22, 127-133. https://doi.org/10.4250/jcu.2014.22.3.127

[27] Reant, P., Labrousse, L., Lafitte, S., et al. (2007) Experimental Validation of Circumferential, Longitudinal, and Radial 2-Dimensional Strain during Dobutamine Stress Echocardiography in Ischemic Conditions.

[28] Ryo, K., Tanaka, H., Kaneko, A., Fukuda, Y., Onishi, T., Kawai, H., et al. (2012) Efficacy of Longitudinal Speckle Tracking Strain in Conjunction with Isometric Handgrip Stress Test for Detection of Ischemic Myocardial Segments. Echocardiography, 29, 411 .

[29] Wierzbowska-Drabik, K., Hamala, P., Roszczyk, N., Lipiec, P., Plewka, M., et al. (2014) Feasibility and Correlation of Standard 2D Speckle Tracking Echocardiography and Automated Function Imaging Derived Parameters of Left Ventricular Function during Dobutamine Stress Test. The International Journal of Cardiovascular Imaging, 30, 729-737. https://doi.org/10.1007/s10554-014-0386-Z

[30] Yu, Y., Villarraga, H.R., Saleh, H.K., Cha, S.S. and Pellikka, P.A. (2013) Can Ischemia and Dyssynchrony Be Detected during Early Stages of Dobutamine Stress Echocardiography by 2-Dimensional Speckle Tracking Echocardiography? The International Journal of Cardiovascular Imaging, 29, 95-102. https://doi.org/10.1007/s10554-012-0074-9

[31] Aggeli, C., Lagoudakou, S., Felekos, I., Panagopoulou, V., et al. (2015) Two-Dimensional Speckle Tracking for the Assessment of Coronary Artery Disease during Dobutamine Stress Echo: Clinical Tool or Merely Research Method. Cardiovascular Ultrasound, 13, 43. https://doi.org/10.1186/s12947-015-0038-z

[32] Valtteri, U., Multti, L., Mikko, P., Mari, W., et al. (2016) Two Dimentional Spickle 
Tracking during Dobutamine Stress Echocardiography in Detection of Myocardial Ischemia in Patient with Suspected Coronary Artery Disease. Journal of the American Society of Echocardiography, 29, 470-479.

https://doi.org/10.1016/j.echo.2015.12.013 